\title{
MiR-338-3p Enhances Ovarian Cancer Cell Sensitivity to Cisplatin by Downregulating WNT2B
}

\author{
Qin Niu, Zhenghong Liu, Jia Gao, and Qiao Wang \\ Department of Oncology, The First People's Hospital of Lianyungang, Lianyungang, China.
}

\begin{abstract}
Purpose: Chemoresistance is a concern in ovarian cancer patients, in whom survival remains. MicroRNA, a novel class of small RNAs, have frequently been found to be dysregulated in human malignancies and to act as negative regulators of gene expression. This study aimed to explore the function of miR-338-3p in cisplatin resistance in ovarian cancer and potential molecular mechanisms thereof.

Materials and Methods: The expression levels of miR-338-3p and WNT2B in ovarian cancer tissues and cells were estimated by real-time quantitative polymerase chain reaction (RT-qPCR). In addition, 3-(4,5-dimethylthiazol-2-yl)-2,5-diphenyl-2H-tetrazol3-ium bromide (MTT), transwell, and flow cytometry assays were used to assess biological role of miR-338-3p in vitro. Western blot assay was conducted to measure protein expression of WNT2B, epithelial-mesenchymal transition (EMT)-related proteins, and apoptosis-related proteins. The relationship between miR-338-3p and WNT2B was confirmed by dual-luciferase reporter. Finally, a xenograft tumor model was developed to explore the effects of overexpression of miR-338-3p on tumor growth in ovarian cancer in vivo.

Results: MiR-338-3p was downregulated in cisplatin resistant ovarian cancer tissues and cells. Mechanistically, high expression of miR-338-3p enhanced cell sensitivity to cisplatin by inhibiting proliferation, motility, and EMT and by promoting apoptosis via targeting WNT2B expression in vitro. Furthermore, overexpression of miR-338-3p increased cisplatin sensitivity among ovarian cancer in an in vivo xenograft tumor model.

Conclusion: MiR-338-3p enhances the sensitivity of ovarian cancer cells to cisplatin by downregulating WNT2B.
\end{abstract}

Key Words: WNT2B, miR-338-3p, ovarian cancer, chemoresistance

\section{INTRODUCTION}

According to the statistics, it has been reported that there were more than 14000 ovarian cancer-related deaths and 20000 new cases in 2018 in the United States. ${ }^{1}$ Although surgery and chemotherapy for ovarian cancer have greatly improved in recent years, ${ }^{2}$ overall survival remains less than satisfactory. Af-

Received: July 23, 2019 Revised: September 26, 2019

Accepted: October 16, 2019

Corresponding author: Zhenghong Liu, MD, Department of Oncology, The First People's Hospital of Lianyungang, No.6 Zhenhua East Road, Haizhou District, Lianyungang 222000, Jiangsu, China.

Tel: 86-189-18961322010, E-mail: lyg82072010@163.com

-The authors have no potential conflicts of interest to disclose.

(c) Copyright: Yonsei University College of Medicine 2019

This is an Open Access article distributed under the terms of the Creative Commons Attribution Non-Commercial License (https://creativecommons.org/licenses/ by-nc/4.0) which permits unrestricted non-commercial use, distribution, and reproduction in any medium, provided the original work is properly cited. ter initial chemotherapy therapeutics, patients with advanced ovarian cancer often experience recurrence and die from chemoresistance disease. ${ }^{3}$ Tumor invasion, metastasis, and multidrug resistance have been found to be factors affecting tumor recurrence in ovarian cancer patients. ${ }^{4}$

The development of chemoresistance is becoming a major barrier to the treatment of cancer patients. ${ }^{5}$ Ovarian cancer patients can show poor responses to current treatment with cisplatin, a chemotherapeutic agent for long-term clinical application. It is imperative to investigate an effective therapeutic target than could enhance sensitivity to cisplatin and to elucidate related molecular pathways in order to develop promising therapy strategies for ovarian cancer with cisplatin resistance. Steg, et al. ${ }^{6}$ showed that ovarian cancer stem cells are involved in chemoresistance, metastasis, and tumor recurrence. Ricci, et al. ${ }^{7}$ reported that epithelial-mesenchymal transition (EMT) in ovarian cancer cells was associated with chemoresistance. Migration, attachment to mesothelial cells, and invasion into 
subperitoneal tissue were found to be involved in the formation of metastasis in peritoneal dissemination of gastric cancer. ${ }^{8}$ Chaffer and Weinberg ${ }^{9}$ also reported that EMT can modify the metastatic ability of cancer cells. Singh and Settleman ${ }^{10}$ agreed with the above conclusions that EMT of tumor cells increases metastasis and further indicated that it contributes to drug resistance. In addition, serial analyses of gene expression profiling have revealed that many EMT-related transcription factors are elevated in cisplatin-resistant cells. ${ }^{11}$

MiRNAs, consisting of 21-25 nucleotides, function as guide molecules in RNA silencing. ${ }^{12}$ Research has indicated that miRNA is associated with ovarian cancer drug resistance and can act as prognostic tools for assessing clinical outcomes. ${ }^{13}$ Furthermore, increasing evidence has shown that miR-338-3p acts as a tumor suppressor blocking the growth of thyroid cell by the targeting protein kinase $\mathrm{B} \gamma \cdot{ }^{14} \mathrm{MiR}-338-3 \mathrm{p}$ could inhibit proliferation, migration, invasion, and EMT in osteosarcoma cells by targeting activator of $90 \mathrm{kDa}$ heat shock protein ATPase homolog $1 .{ }^{15}$ Meanwhile, Sun, et al. ${ }^{16}$ showed that miR-338-3p functions as a tumor inhibitor in gastric cancer by targeting protein-tyrosine phosphatase $1 \mathrm{~B}$. Consistent with the above findings, Liu, et al. ${ }^{17}$ demonstrated that miR-338-3p acts as a suppressor of epithelial ovarian cancer progression, although the mechanisms of drug resistance in cancer are poorly understood. In this study, we investigated the expression of miR338-3p in cisplatin-resistant ovarian cancer tissues and cells. Functional and mechanism analyses were conducted to further clarify the relationships among miR-338-3p, WNT2B, and resistance to cisplatin in ovarian cancer.

\section{MATERIALS AND METHODS}

\section{Clinical samples}

In total, 54 frozen human primary ovarian tumor samples were divided into two groups according to their responses to the cisplatin: 24 treatment-responsive patients and 30 treatmentresistant patients, as well as normal non-tumors tissues, were collected from The First People's Hospital of Lianyungang. Biopsy samples were promptly frozen in liquid nitrogen and maintained at $-80^{\circ} \mathrm{C}$ until ready for further use. This study was approved by the Ethical Committee of The First People's Hospital of Lianyungang, and written informed consent was obtained from all participants before surgery. All biopsy samples used in this study were histopathologically examined by two independent pathologists.

\section{Cells culture and transfection}

SKOV3 cells (human ovarian carcinoma cell line) were purchased from the American Type Culture Collection (Manassas, VA, USA), and A2780 (human ovarian carcinoma cell line) and IOSE-80 (human ovarian epithelial cell line) cells were obtained from the Cancer Research Institute, Southern Medi- cal University (Guangzhou, China). Cells were cultured in RPMI-1640 medium (GIBCO BRL, Grand Island, NY, USA) with $10 \%(\mathrm{v} / \mathrm{v})$ fetal bovine serum (GIBCO BRL) in a $5 \% \mathrm{CO}_{2}$ atmosphere at $37^{\circ} \mathrm{C}$. Matched cisplatin-resistant ovarian cancer cells, named SKOV3/DDP and A2780/DDP cells, were produced from the parental cell lines and cultured with escalating concentrations of cisplatin as previously described. ${ }^{18}$ Overexpressed plasmids of miR-338-3p (miR-338-3p) and its negative control miR-NC, specific short hairpin RNA (shRNA) against WNT2B (sh-WNT2B) and its negative control sh-NC, and overexpressed plasmids of WNT2B (WNT2B) and its negative control pcDNA were purchased from RiboBio (Guangzhou, China). The above oligonucleotides or plasmids were transfected into ovarian cancer cell lines utilizing Lipofectamine 2000 reagent (Thermo Fisher Scientific, Waltham, MA, USA) according to the manufacturer's instructions.

\section{RNA isolation and real-time quantitative polymerase chain reaction}

Total RNA from cells and tissues was extracted with TRIzol reagent (Thermo Fisher Scientific). The quality of samples was regard as good when OD260 nm/OD280 $\mathrm{nm}$ values were greater than 1.8. The Prime Script RT Reagent kit (Takara, Dalian, China) and microRNA Reverse Transcription kit (Thermo Fisher Scientific) were employed to reverse transcribe RNA into complementary DNA. The expression levels of miR-338-3p and WNT2B were analyzed using SYBR Premix Ex Taq II (Takara) for real-time quantitative polymerase chain reaction (RT-qP$\mathrm{CR}$ ) on an ABI 7500 HT system (Applied Biosystems, Foster City, CA, USA). Relatively quantitative analysis was performed for mean values using the $2^{-\Delta \Delta C t}$ method. Glyceraldehyde3-phosphate dehydrogenase (GAPDH) or endogenous small nuclear RNA U6 served as an internal control.

The following primers were utilized: miR-338-3p (forward, 5'-ATCCAGTGCGTGTCGTGG-3'; reverse, 5'-TGCTTCCAGC ATCAGTGAT-3'); WNT2B (forward, 5'-ATTTCCCGCTCTGG AGATTT-3'; reverse, 5'-AAGCTGGTGCAAAGGAAAGA-3'); GAPDH (forward, 5'-TCCCATCACCATCTTCCAGG-3'; reverse, 5'- GATGACCCTTTTGGCTCCC-3'); and U6 (forward, 5'-CTCGCTTCGGCAGCACA-3'; reverse, 5'-AACGCTTCACG AATTTGCGT-3').

\section{MTT assay}

Cell viability was detected with 3-(4, 5-dimethylthiazol-2-yl)-2, 5-diphenyl-2H-tetrazol-3-ium bromide (MTT) assay. Firstly, cells were seed onto 96 -well plates at a density of $5.0 \times 10^{3}$ cells per well and treated with various doses of cisplatin for $48 \mathrm{~h}$. After this, $20 \mu \mathrm{L}$ of $5 \mathrm{mg} / \mathrm{mL}$ MTT (Sigma-Aldrich Co., St. Louis, MO, USA) was added to each well and incubated at $37^{\circ} \mathrm{C}$ for another $4 \mathrm{~h}$. Formazan crystals were then dissolved with dimethyl sulfoxide (DMSO). Absorbance at a wavelength of 490 nm was measured using a microplate reader (Applied Biosystems). Relative survival curves were drawn to determine $\mathrm{IC}_{50}$ 
values for the dose of cisplatin causing $50 \%$ inhibition of growth among ovarian cancer cells.

\section{Flow cytometry analysis of apoptosis}

SKOV3/DDP and A2780/DDP cells $\left(3.5 \times 10^{5} /\right.$ well $)$ after transfection were seeded into a 6 -well plate treated with various doses of cisplatin for $48 \mathrm{~h} .500 \mu \mathrm{L}$ of binding buffer was then added to each well. Subsequently, $5 \mu \mathrm{L}$ of propodeum iodide (Thermo Fisher Scientific) and $5 \mu \mathrm{L}$ of Annexin V-Fluorescein isothiocyanate (Thermo Fisher Scientific) were added at room temperature for $15 \mathrm{~min}$. The ratio of apoptotic cells was detected using a flow cytometer (Applied Biosystems).

\section{Western blot assay}

Total protein was subjected to sodium dodecyl sulfate polyacrylamide gel electrophoresis (SDS-PAGE) and transferred to polyvinylidene fluoride (PVDF) membranes (Millipore, Billerica, MA, USA). Subsequently, membranes were blocked with 5\% nonfat milk before incubation with 1:2000 dilution primary antibody at $4^{\circ} \mathrm{C}$ overnight, and then the membranes were incubated with secondary antibody with horseradish peroxidase for $2 \mathrm{~h}$ at a 1:5000 dilution. Finally, the signal intensities of antigen-antibody complexes were visualized using chemiluminescence.

\section{Cell migration and invasion assay}

For invasion assay, cells were serum starved for $24 \mathrm{~h}$ prior to use and were placed into 24-well plates with a polycarbonate membrane with 8 - $\mu$ m pores (Thermo Fisher Scientific) and Matrigel (Thermo Fisher Scientific) at $5 \times 10^{4}$ cells/well. Lower chambers containing fetal bovine serum were used to induce cells through membranes. Plates were incubated for $24 \mathrm{~h}$ at $37^{\circ} \mathrm{C}$ in $5 \% \mathrm{CO}_{2}$. After $24 \mathrm{~h}$, the average number of invaded cells was calculated in five random fields of view under a microscope (Applied Biosystems) to produce a diagram of invasion cell numbers. Similarly, migration assays were conducted as described above, except the use of Matrigel was omitted.

\section{Dual-luciferase reporter assay}

The 3'UTR segments of WNT2B, which were predicted to interact with miR-338-3p using starBase2.0 (http://starbase.sysu. edu.cn/starbase2/index.php), were amplified by PCR and cloned downstream of the Renilla luciferase stop codon in pGL3 vector (Thermo Fisher Scientific), named WT or MUT reporters, respectively. Ovarian cancer cells were infected with reporter vectors using Lipofectamine 2000 (Thermo Fisher Scientific) in accordance with the provided instruction manual. Cells were harvested at 24 -h post-transfection, and firefly or Renilla fluorescence signals were read using the Dual-Luciferase Reporter Assay System (Thermo Fisher Scientific). Renilla luciferase served as an internal control. Three independent experiments were performed.

\section{In vivo experiment}

In total, 21 male BALB/c nude mice were purchased from Shanghai Experimental Animal Center (Shanghai, China) and fed according to institutional guidelines. SKOV3/DDP cells transfected with miR-NC or miR-338-3p were subcutaneously injected into the right backs of the nude mice. The mice were treated with PBS or cisplatin $(5.0 \mathrm{mg} / \mathrm{kg}$ ) with intraperitoneally injected for one cycle of three days at 7-d post-transfection. Tumor growth was monitored every week, and size was calculated using $\mathrm{V}=1 / 2 \times \mathrm{ab}^{2}$ [maximum (a) and minimum (b) length of the tumor]. At $35 \mathrm{~d}$ after injection, mice were sacrificed, and tumor samples were weighed and analyzed by Western blot and RT-qPCR assays. Animal experiments were approved by the Institutional Animal Care and Use Committee of The First People's Hospital of Lianyungang.

\section{Statistical analysis}

All statistical analyses were conducted with GraphPad Prism 7 (GraphPad Inc, La Jolla, CA, USA), and results are expressed as mean \pm standard deviation. $p$ values less than 0.05 were regard as statistically significant. Differences between two groups were analyzed by Student's t-test. One-way analysis of variance was used to estimate differences among three or more groups.

\section{RESULTS}

\section{MiR-338-3p expression is low in cisplatin-resistant ovarian cancer tissues and cells}

To begin, RT-qPCR was performed to detect the expression of miR-338-3p in normal tissues and ovarian cancer tissues (DDPsensitive/DDP-resistance), as well as in IOSE-80, A2780, and SKOV3 cells and cisplatin-resistant cells (A2780/DDP and SKOV3/DDP cells). As presented in Fig. 1A and B, the data showed that miR-338-3p was significantly downregulated in ovarian cancer tissues and cells, compared with matched controls. Moreover, a marked decrease in miR-338-3p expression was observed in cisplatin-resistant tissues and cells, compared with their cisplatin-sensitive counterparts. Kaplan-Meier analysis with log-rank test further showed that low expression levels of miR-338-3p led to a lower survival rate of ovarian cancer patients, while high expression of miR-338-3p facilitated a higher survival rate (Fig. 1C). We concluded that expression of miR-338-3p is associated with cisplatin resistance in ovarian cancer patients.

\section{Overexpression of miR-338-3p enhances sensitivity to cisplatin in ovarian cancer cells \\ Gain-of-function experiments confirmed that the expression levels of miR-338-3p were increased in cisplatin-resistant ovar- ian cancer cells transfected with miR-338-3p mimic (Fig. 2A). The $\mathrm{IC}_{50}$ values of cisplatin in A2780/DDP and SKOV3/DDP cells transfected with miR-338-3p were lower than those in}



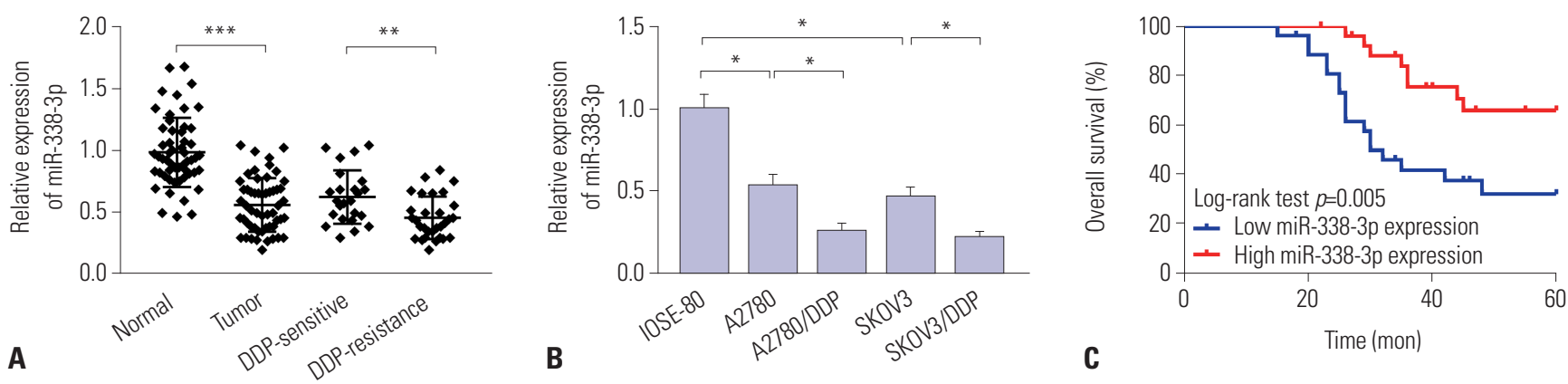

Fig. 1. Expression of miR-338-3p in ovarian cancer tissues and cell clines. (A) Real-time quantitative polymerase chain reaction (RT-qPCR) assay was performed to examine the expression of miR-338-3p in 54 ovarian cancer tissues (divided into two groups according to resistance to cisplatin) and adjacent normal tissues. (B) The expression levels of miR-338-3p in human normal ovarian cancer cells (IOSE-80) and ovarian cancer cells (A2780 and SKOV3), as well as cisplatin resistant ovarian cancer cells (A2780/DDP and SKOV3/DDP), were assessed by RT-qPCR. (C) The overall survival curve of ovarian cancer patients was analyzed with Kaplan-Meier analysis. ${ }^{*} p<0.05 ;{ }^{* *} p<0.01 ;{ }^{* * *} p<0.001$.

miR-NC groups (Fig. 2B and C). To further explore the proliferative ability of A2780/DDP and SKOV3/DDP cells transfected with miR-NC or miR-338-3p, MTT assay indicated that high expression of miR-338-3p repressed the proliferation of ovarian cancer cells (Fig. 2D and E). In addition, overexpression of miR-338-3p elicited a higher apoptosis rate in miR-338-3p groups than that in miR-NC groups (Fig. 2F). Changes in apoptosis-related proteins were consistent with changes in apoptotic rate: upregulation of miR-338-3p promoted the expressions of cleaved-caspase- 3 and Bax, but inhibited the expression of Bcl-2 (Fig. 2G and H). Transwell migration and invasion assays showed that upregulation of miR-338-3p inhibited migration and invasion of A2780/DDP and SKOV3/DDP cells (Fig. 2I and J). Investigation of the effects of miR-338-3p overexpression on the protein levels of E-cadherin, $\mathrm{N}$-cadherin, and Vimentin by Western blot assay showed that protein levels of $\mathrm{N}$ cadherin and Vimentin were remarkably reduced, while Ecadherin levels increased in response to transfection of miR338-3p into A2780/DDP and SKOV3/DDP cells (Fig. 2K and L). Furthermore, levels of alpha smooth muscle actin ( $\alpha$-SMA) and fibronectin were mitigated in A2780/DDP and SKOV3/DDP cells transfected with miR-338-3p mimic (Supplementary Fig. 1A, only online). Collectively, miR-338-3p enhanced the sensitivity of ovarian cancer cells to cisplatin.

\section{MiR-338-3p negatively targets WNT2B}

WNT2B and its mutant sites are shown in Fig. 3A. Additionally, the results of dual-luciferase reporter assay revealed that elevated expression of miR-338-3p significantly repressed the luciferase activity of WNT2B 3'UTR-WT reporter, but not that of WNT2B 3'UTR-MUT reporter in A2780/DDP and SKOV3/DDP cells (Fig. 3B and C). To further clarify the expression levels of WNT2B in ovarian cancer cells and tissues, the protein and mRNA levels of WNT2B were assessed in treatment-responsive patient tissues or cells (A2780 and SKOV3) and treatmentresistant patient tissues and cells (A2780/DDP and SKOV3/ DDP), as well as matched normal controls, utilizing RT-qPCR and Western blot assays. We discovered that the expression levels of WNT2B in cisplatin-resistant ovarian cancer cells (A2780/DDP and SKOV3/DDP) or tissues were markedly increased (Fig. 3D-F). Furthermore, a negative correlation between WNT2B and miR-338-3p was confirmed by RT-qPCR assay (Fig. 3G). Subsequently, we assessed the protein and mRNA levels of WNT2B in A2780/DDP and SKOV3/DDP cells introduced with miR-NC, miR-338-3p, miR-338-3p+pcDNA, or miR-338-3p+WNT2B. As shown in Fig. 3H-K, miR-338-3p overexpression markedly inhibited the expression levels of WNT2B at both the protein and mRNA level; meanwhile, reintroducing WNT2B could attenuate miR-338-3p-induced reductions in WNT2B. Taken together, these data demonstrated that miR-338-3p targets WNT2B expression.

\section{WNT2B knockdown sensitizes A2780/DDP and SKOV3/ DDP cells to cisplatin}

RT-qPCR and Western blot assays indicated that WNT2B expression is reduced in A2780/DDP and SKOV3/DDP cells transfected with sh-WNT2B in comparison with matched control (Fig. 4A and B). Cells were treated with different doses of cisplatin for $48 \mathrm{~h}$, and then $\mathrm{IC}_{50}$ values were detected by MTT assay. The results showed that WNT2B knockdown significantly decreased cisplatin resistance among A2780/DDP and SKOV38/DDP cells (Fig. 4C and D). Cell viability determination results showed that WNT2B knockdown significantly inhibited proliferation of A2780/DDP and SKOV3/DDP cells (Fig. 4E and F). Flow cytometry assay showed that apoptosis rates increased in SA2780/DDP and SKOV3/DDP cells after treatment with shWNT2B (Fig. 4G). Assessment of apoptosis-related proteins by Western blot assay revealed that decreased expression of WNT2B promotes the expression of cleaved-caspase- 3 and Bax, but impedes the expression of Bcl-2 (Fig. 4H and I). Migration and invasion assays were carried out to evaluate motility ability of ovarian cancer cells. The results suggested that downregulation of WNT2B suppresses migration and invasion of cisplatin-resistance cells (Fig. 4J and K). Analogously, WNT2B silencing suppressed EMT processes in ovarian cancer cells by reducing $\mathrm{N}$-cadherin and Vimentin expression, 


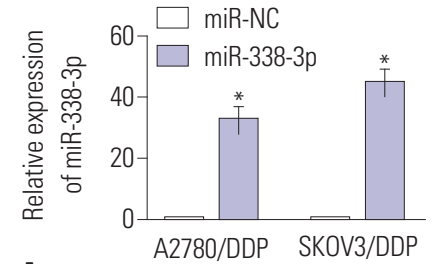

A
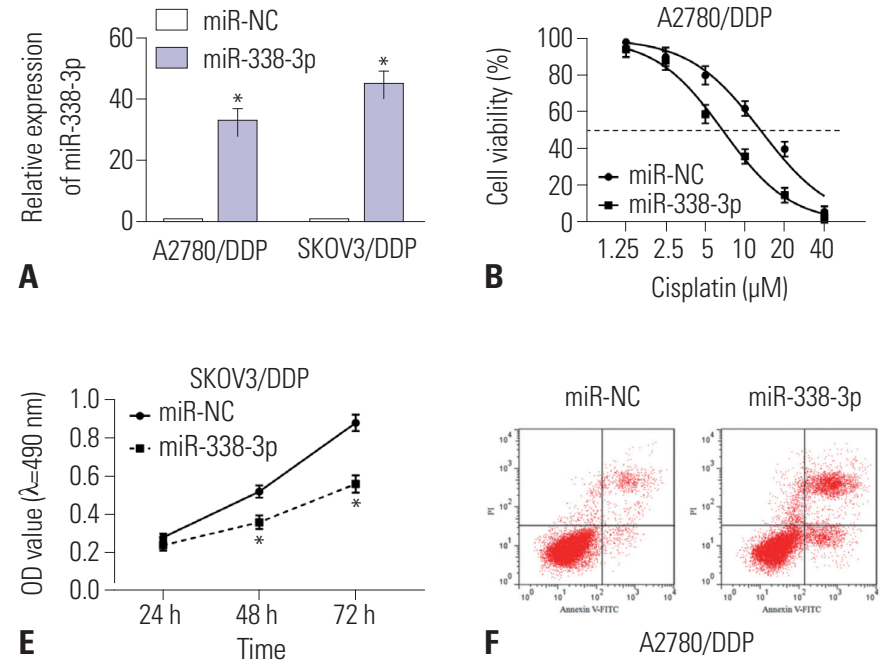

A2780/DDP

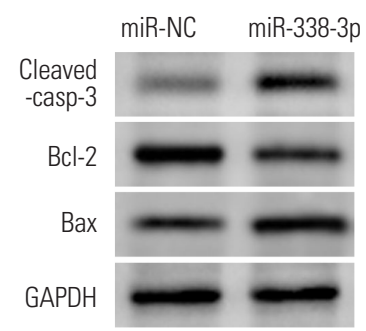

G

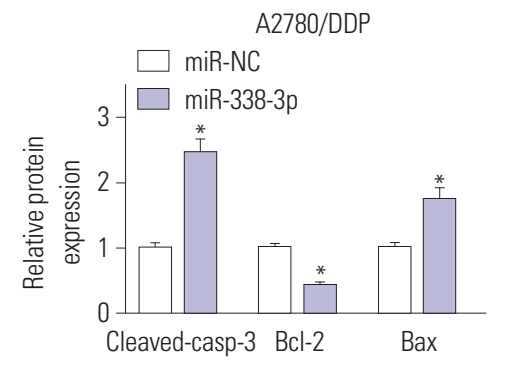

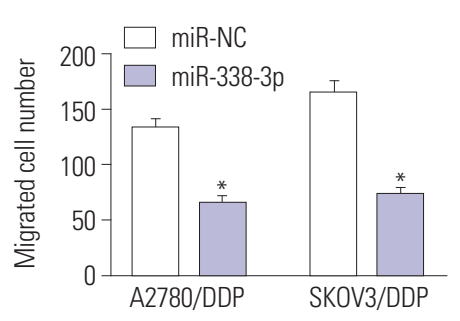

I
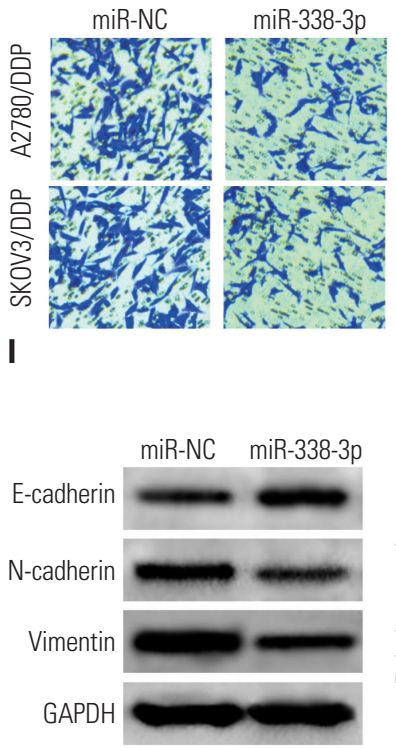

K

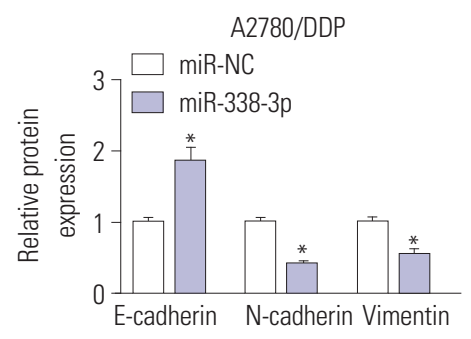

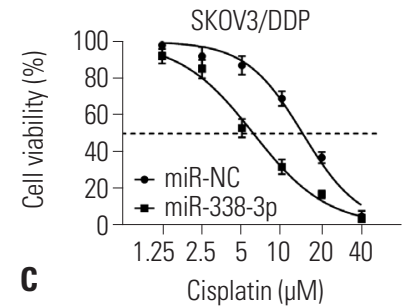
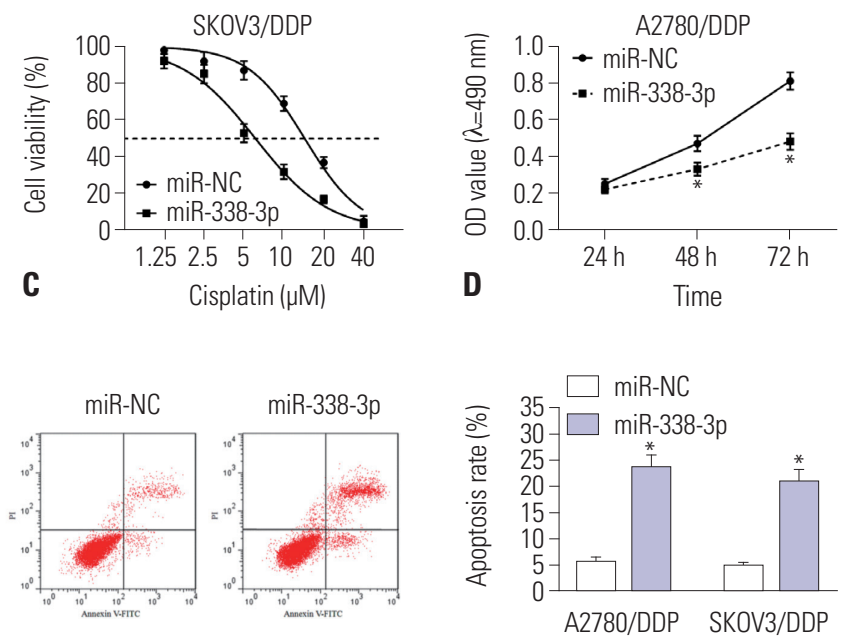

SKOV3/DDP
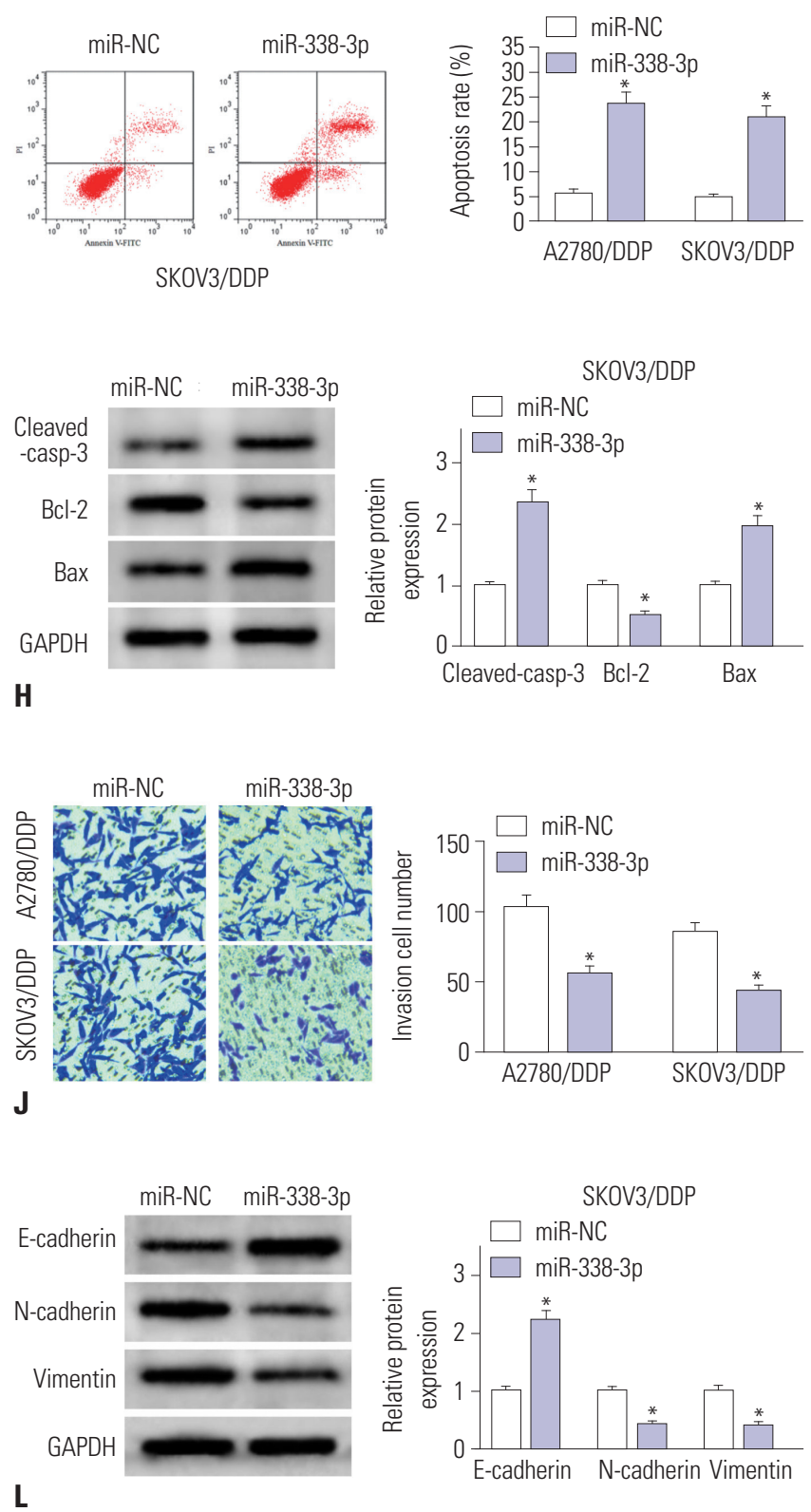

Fig. 2. Increased expression of miR-338-3p enhances sensitivity to cisplatin among ovarian cancer cells. A2780/DDP and SKOV3/DDP cells were transfected with miR-NC or miR-338-3p. (A) The relative expression levels of miR-338-3p in transfected cisplatin-resistant ovarian cancer cells was evaluated via real-time quantitative polymerase chain reaction (RT-qPCR). (B-E) IC 50 $_{10}$ values of cisplatin, as well as cell viability in A2780/DDP and SKOV3/DDP cells, were measured by MTT assay. (F) Apoptotic rate was assessed by flow cytometry in A2780/DDP and SKOV3/ DDP cells after transfection. (G and H) Protein levels of cleaved-caspase-3, Bcl-2, and Bax were determined in A2780/DDP and SKOV3/DDP cells by Western blot. (I and J) Cell migration and invasion were evaluated in transfected A2780/DDP and SKOV3/DDP by transwell assay ( $\times 100$, stained with $0.1 \%$ crystal violet). (K and L) Western blot assay was used to assess the expression levels of epithelial-mesenchymal transition-related proteins (E-cadherin, $\mathrm{N}$-cadherin and Vimentin). . $p<0.05$. GAPDH, glyceraldehyde-3-phosphate dehydrogenase. 


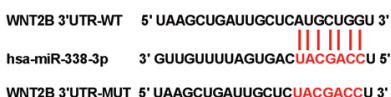

A
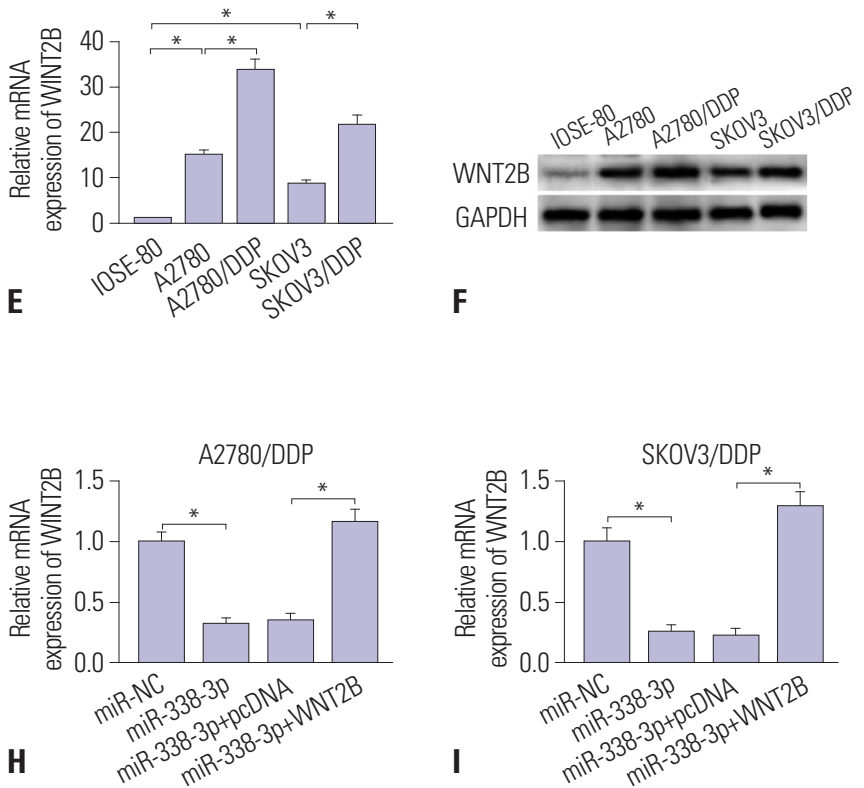
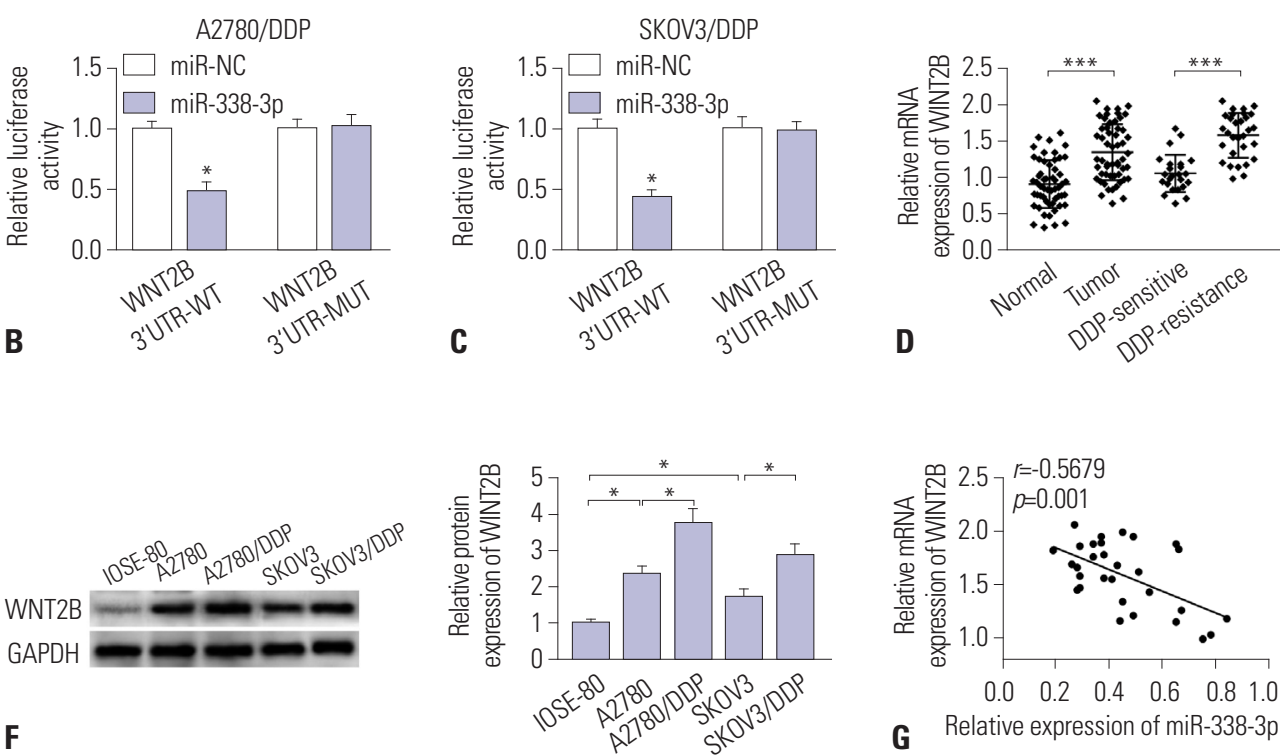

G

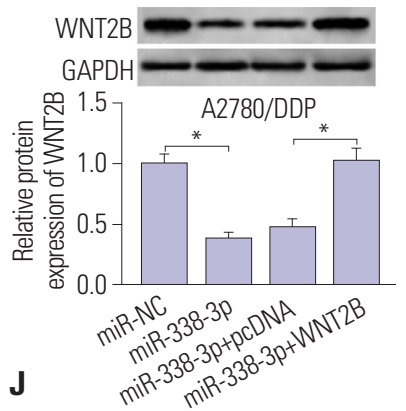

Fig. 3. WNT2B is a target of miR-338-3p. (A) The binding sites of miR-338-3p on the $3^{\prime} U T R$ of WNT2B was predicted with bioinformatics and its mutant are shown. (B and C) Luciferase activity was estimated in A2780/DDP and SKOV3/DDP co-transfected with WNT2B 3'UTR-WT/MUT luciferase reporters and miR-338-3p/miR-NC. (D-F) The protein and mRNA levels of WNT2B were measured in treatment-responsive patient tissues or cells (A2780 and SKOV3) and treatment-resistant patients tissues and cells (A2780/DDP and SKOV3/DDP), as well as matched normal controls, utilizing real-time quantitative polymerase chain reaction (RT-qPCR) and Western blot assay. (G) The relationship between miR-338-3p and WNT2B was analyzed. (H-K) Gain-of-function experiments were performed to detect mRNA and protein expression levels of WNT2B in A2780/DDP and SKOV3/DDP cells transfected with miR-NC, miR-338-3p, miR$338-3 p+p c$ DNAN or miR-338-3p+WNT2B. * $p<0.05 ;{ }^{* * *} p<0.001$. GAPDH, glyceraldehyde-3-phosphate dehydrogenase.

while promoting E-cadherin (Fig. 4L and M). Meanwhile, consistent with $\mathrm{N}$-cadherin and Vimentin, $\alpha$-SMA and fibronectin were inhibited by sh-WNT2B in A2780/DDP and SKOV3/DDP cells (Supplementary Fig. 1B, only online). Collectively, these data suggested that WNT2B knockdown weakens resistance to cisplatin among A2780/DDP and SKOV3/DDP cells.

\section{MiR-338-3p impedes cisplatin resistance in ovarian cancer cells by downregulating WNT2B expression} A2780/DDP and SKOV3-8/DDP were transfected with miRNC, miR-338-3p, miR-338-3p+pcDNA, or miR-338-3p+WNT2B. The transfected A2780/DDP and SKOV3/DDP cells were treated with various doses of cisplatin for $48 \mathrm{~h}$, and $\mathrm{IC}_{50}$ values of cisplatin were measured by MTT assay. The results thereof showed that gain of WNT2B overturned upregulation of miR142-5p-mediated resistance to cisplatin in ovarian cancer cells (Fig. 5A and B). Furthermore, we also found that greater expression of WNT2B reversed the reduced viability in A2780/
DDP and SKOV3/DDP cells caused by miR-338-3p overexpression (Fig. 5C and D). As shown in Fig. 5E, upregulation of miR$338-3 p$ resulted in a promotion of apoptosis in A2780/DDP and SKOV3/DDP cells, which was suppressed by overexpression of WNT2B, as demonstrated by flow cytometry analysis. Western blot assay was conducted to detect the protein expression levels of transfected cells and indicated that miR-338-3p overexpression markedly suppressed anti-apoptosis protein levels (Bcl-2) and enhanced pro-apoptosis protein levels (cleavedcaspase-3 and Bax). Reversal of WNT2B expression abolished the observed miR-338-3p-induced effects (Fig. 5F and G). Consistent with proliferation, overexpression of WNT2B abolished inhibition effects of miR-338-3p on migration and invasion in resistant ovarian cancer cells (Fig. 5H and I). Analogously, the protein levels of $\mathrm{N}$-cadherin and Vimentin were apparently reduced, while E-cadherin was enhanced, in the miR-338-3p overexpression group, although upregulation of WNT2B overturned this effect (Fig. 5J and K). In addition, repression of $\alpha$-SMA 
and fibronectin by treatment with miR-338-3p were abated in A2780/DDP and SKOV3/DDP cells by combining with WNT2B (Supplementary Fig. 1C, only online). Taken together, we determined that miR-338-3p directly targets WNT2B to reduce resistance in ovarian cancer cells.

\section{Elevated miR-338-3p suppresses tumor growth in vivo} As presented in Fig. 6A and B, cisplatin treatment significantly decreased tumor volume and weight, while miR-338-3p+cisplatin treatment led to more obvious inhibition effects on the volumes and weights of tumors, compared to other groups. The expression levels of miR-338-3p were markedly higher in tumor tissues of the emiR-338-3p+cisplatin group than the other groups (cisplatin+miR-NC or miR-NC group); moreover, mRNA expression levels of WNT2B decreased in tumor tissues from the miR-338-3p+cisplatin group, compared with other groups (cisplatin+miR-NC or in miR-NC group) (Fig. 6C). In addition, overexpression of miR-338-3p induced a decrease in protein
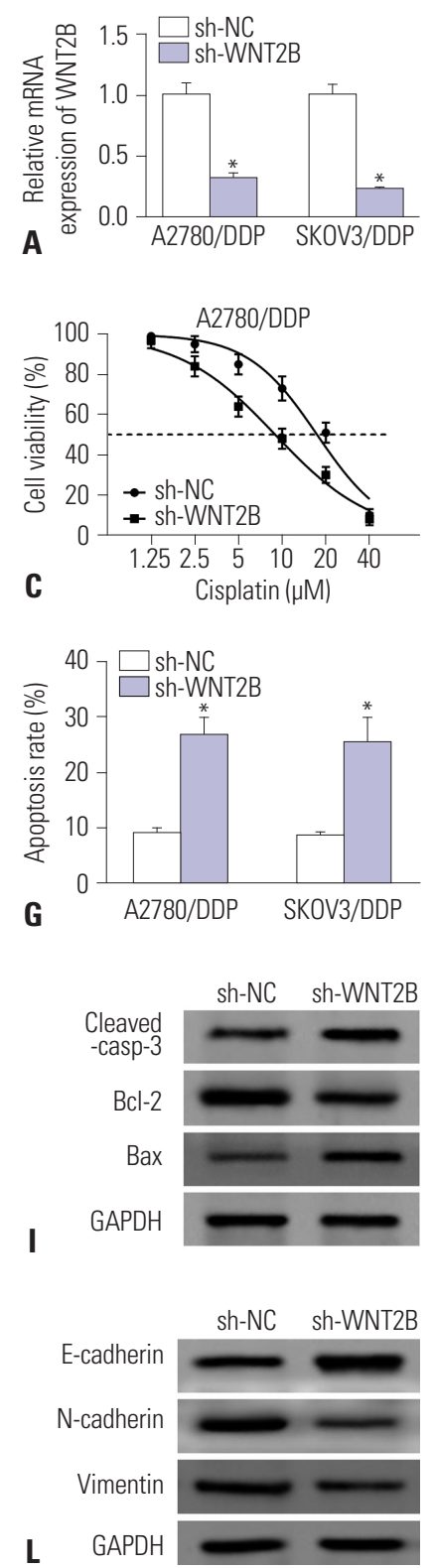
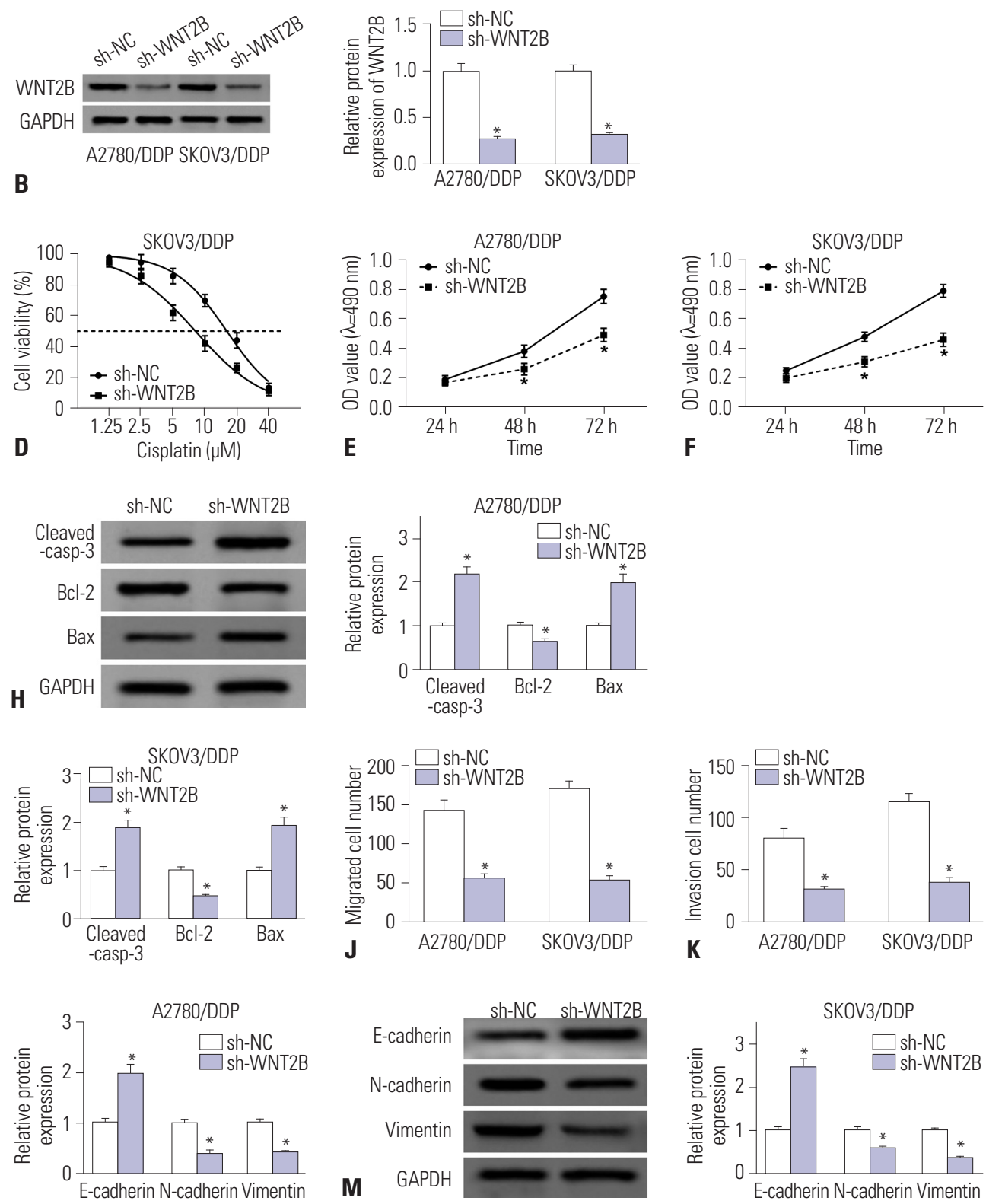

Fig. 4. WNT2B knockdown weakens resistance to cisplatin in ovarian cancer cells. A2780/DDP and SKOV3/DDP cells were transfected with sh-NC or shWNT2B. (A and B) real-time quantitative polymerase chain reaction (RT-qPCR) and Western blot assays were carried out to evaluate expression levels of WNT2B in transfected ovarian cancer cells. (C-F) MTT analysis was utilized to assess $I_{50}$ values for cisplatin and cell viability in ovarian cancer cells post-transfection. (G) Apoptosis of transfected ovarian cancer cells was determined by flow cytometry. (H and I) Levels of apoptosis-related proteins (cleaved-caspase-3, Bcl-2, and Bax) were measured with Western blot. ( $\mathrm{J}$ and $\mathrm{K}$ ) Transwell assay was performed to detect the motility of ovarian cancer cells. (L and M) Western blot was used to assess the expression levels of E-cadherin, N-cadherin, and Vimentin. ${ }^{*} p<0.05$. GAPDH, glyceraldehyde3-phosphate dehydrogenase. 
expression of WNT2B in tumor tissues (Fig. 6D).These results demonstrated that miR-338-3p represses tumor growth in vivo by affecting WNT2B expression.

\section{DISCUSSION}

In the present study, we found the expression levels of miR-338$3 p$ to be associated with chemoresistance to cisplatin in ovarian cancer patients. Functional and mechanical experiments
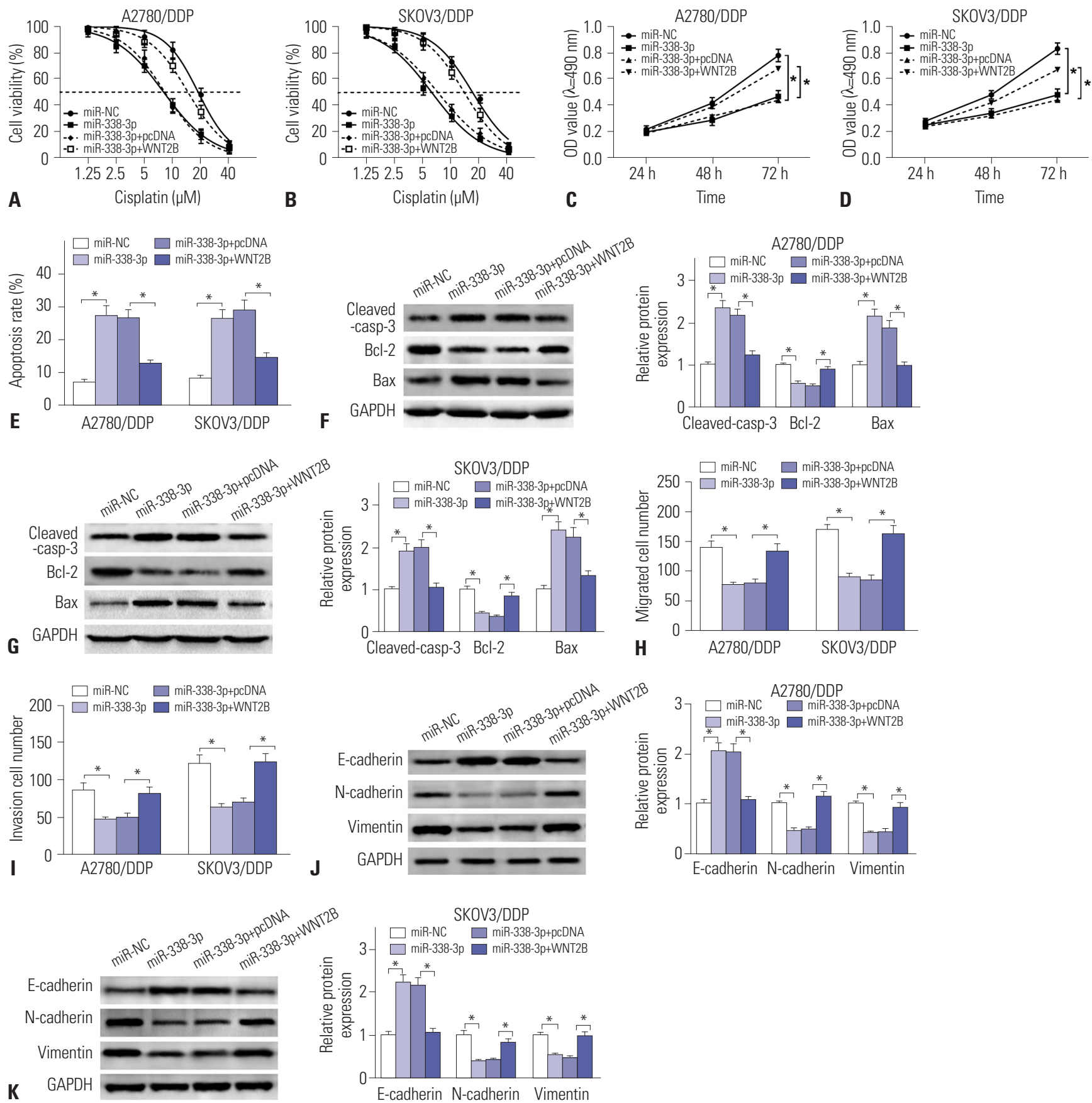

Fig. 5. Upregulation of miR-338-3p increases cisplatin sensitivity in resistant ovarian cancer cells by negatively regulating WNT2B. A2780/DDP and SKOV3/

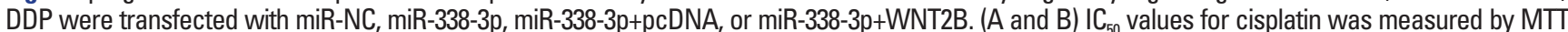
assay in transfected A2780/DDP and SKOV3/DDP cells treated with various doses of cisplatin for $48 \mathrm{~h}$. (C and D) MTT analysis was used to measure the viability of transfected A2780/DDP and SKOV3/DDP cells. (E) Apoptosis rates of 2780/DDP and SKOV3/DDP cells were detected by flow cytometry assay. ( $F$ and G) Western blot assay was conducted to test the protein expression levels of apoptosis-related proteins in transfected cells. (H and I) The migration and invasion capabilities in transfected ovarian cancer cells were measured by transwell assay. ( $\mathrm{J}$ and $\mathrm{K}$ ) The protein expression levels of epithelialmesenchymal transition-related in transfected A2780/DDP and SKOV3/DDP cells were detected by Western blot assay. ${ }^{*} p<0.05$. GAPDH, glyceraldehyde3-phosphate dehydrogenase. 


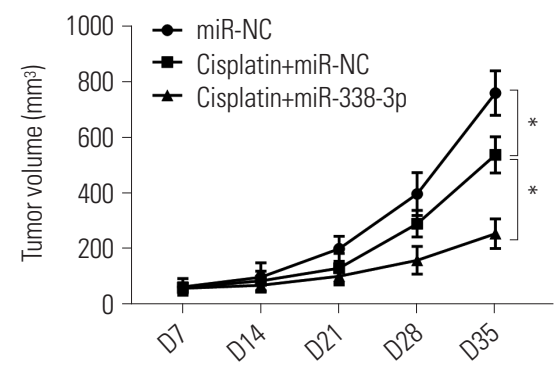

A

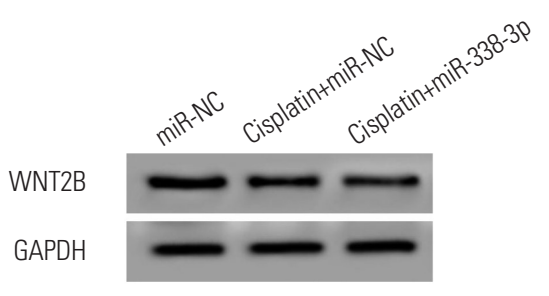

D

Fig. 6. Elevated expression of miR-338-3p suppresses tumor growth and enhances cisplatin sensitivity by affecting WNT2B expression in vivo. (A and B) Growth curves for tumor volume and weight were measured in xenograft tumors from nude mice treated with PBS or cisplatin $(5.0 \mathrm{mg} / \mathrm{kg}$, once every three days). (C and D) RT-qPCR and Western blot assay were performed to measure the expression levels of miR-338-3p and WNT2B in resected tumor tissues, respectively. * $p<0.05$. GAPDH, glyceraldehyde-3-phosphate dehydrogenase.

further revealed that miR-338-3p enhances sensitivity to cisplatin via downregulating WNT2B in ovarian cancer cells.

Previous study have shown that miRNA-630 inhibitor has a similar function as miR-338-3p and that suppression of miR630 could sensitize SKOV3-TR cells to paclitaxel by increasing apoptosis. ${ }^{19}$ Analogously, miRNA-574-3p could target epidermal growth factor receptor to sensitize ovarian cancer cells to paclitaxel and cisplatin. ${ }^{20} \mathrm{Li}$, et al ${ }^{21}$ indicated that miR-142-5p combined with cisplatin treatment could increase apoptosis in ovarian cancer cells by regualating apoptotic-associated genes expression. In addition, enforced expression of let- 7 has bee found to enhance sensitivity to Taxol or vinblastine in ovarian cancer cells. ${ }^{22}$ Our data further showed that overexpression of miR-338-3p enhances sensitivity to cisplatin among ovarian cancer cells by regulating proliferation, apoptosis, and EMT process. Meanwhile, Zhang, et al. ${ }^{23}$ found that miR-338-3p regulates metabolism in ovarian cancer cell by inhibiting pyruvate kinase M2 expression. In the present study, bioinformatics analysis and dual-luciferase reporter assay were conducted and confirmed that miR-338-3p had putative binding sites on the 3'UTR of WNT2B: While WNT2B, WNT5A, WNT5B, and WNT9B all had putative binding sites with miR-338-3p, only co-transfection wild-type WNT2B and miR-338-3p showed reduced luciferase activity, as presented in Supplementary Fig. 2 (only online). Thus, the function of WNT2B was mainly explored in subsequence experiments. In view of the high expression of WNT2B in cisplatin resistance cells, the function of
WNT2B knockdown in ovarian cancer cells was further explored. WNT2B plays a key role in Wnt/ $\beta$-catenin signal pathway by regulating the proliferation, apoptosis, and EMT of cells. Jiang and $\mathrm{Ma}^{24}$ demonstrated that the expression of miR324-3p in polycystic ovary syndrome rats is remarkably downregulated and confirmed that overexpression of miR-324-3p represses proliferation and induces the apoptosis of granulosa cells by targeting of WNT2B. Moreover, other have found that miR-185-3p regulates radio-resistance of nasopharyngeal carcinoma via targeting WNT2B in vitro. ${ }^{25}$ Furthermore, HOXD cluster antisense RNA 1 promoted proliferation, invasion, and EMT of epithelial ovarian cancer cells has been found to be affected by Wnt/ $\beta$-catenin signaling. ${ }^{26}$ Altogether, the Wnt/ $\beta$-catenin pathway appears to be involved in EMT as a major contributor to the tumorigenesis of ovarian cancer. ${ }^{27}$ In our research, the overexpression of miR-338-3p suppressed EMT by downregulating $\mathrm{N}$-cadherin and Vimentin expression and by increasing E-cadherin. Importantly, upregulation of WNT2B overturned the effects of miR-338-3p overexpression on EMT. In agreement with our data, a review conducted by Du and Shim ${ }^{28}$ confirmed that EMT plays an important role in metastasis and drug resistance among cancer cells. E-cadherin, N-cadherin, and Vimentin are considered EMT-associated marker proteins. Research indicated that E-cadherin is an important molecule for cell-cell adhesion and functions as a biomarker of EMT. Indeed, low expression of E-cadherin has been shown to facilitate separation of cancer cells from the serosal surface..$^{29}$ 
Here, we explored the influence of miR-338-3p on cisplatin resistance in ovarian cancer and confirmed that the expression of miR-338-3p is positively correlated with 5-year survival rate in ovarian cancer patients. We also showed that expression of miR-338-3p enhances the sensitivity of ovarian cancer cells to cisplatin in vivo and in vitro.

Regrettably, our experiment did not conduct a comprehensive study on drug metabolism-related proteins. Our results demonstrated that abundant expression of miR-338-3p suppresses resistance to cisplatin in ovarian cancer cells by affecting WNT2B. In the future research, we will focus on whether the impact effect of miR-338-3p/WNT2B on cisplatin resistance is associated with drug metabolism signaling pathways. Knowing the molecular factors involved in drug resistance may help us to develop new strategies for chemotherapy and reduce the relapse rate of ovarian cancer.

In conclusion, our data suggest that miR-338-3p is involved in cisplatin resistance in ovarian cancer. MiR-338-3p appears to act as a therapeutic target through which to enhance sensitivity of ovarian cancer cells to cisplatin by targeting WNT2B.

\section{AUTHOR CONTRIBUTIONS}

Conceptualization: Zhenghong Liu. Data curation: Jia Gao. Formal analysis: Qin Niu. Funding acquisition: Qiao Wang. Investigation: Zhenghong Liu. Methodology: Qin Niu. Project administration: Zhenghong Liu. Resources: Jia Gao and Qiao Wang. Software: Qiao Wang. Supervision: Zhenghong Liu and Qin Niu. Validation: Qin Niu and Jia Gao. Visualization: Qin Niu and Qiao Wang. Writing_original draft: Qin Niu and Zhenghong Liu. Writing_review \& editing: Qin Niu, Zhenghong Liu, and Qiao Wang.

\section{ORCID iDs}

$\begin{array}{ll}\text { Qin Niu } & \text { https://orcid.org/0000-0001-5832-6685 } \\ \text { Zhenghong Liu } & \text { https://orcid.org/0000-0003-0414-8988 } \\ \text { Jia Gao } & \text { https://orcid.org/0000-0002-0641-4736 } \\ \text { Qiao Wang } & \text { https://orcid.org/0000-0002-8675-5804 }\end{array}$

\section{REFERENCES}

1. Torre LA, Trabert B, DeSantis CE, Miller KD, Samimi G, Runowicz CD, et al. Ovarian cancer statistics, 2018. CA Cancer J Clin 2018;68: 284-96.

2. Ozols RF, Bundy BN, Greer BE, Fowler JM, Clarke-Pearson D, Burger RA, et al. Phase III trial of carboplatin and paclitaxel compared with cisplatin and paclitaxel in patients with optimally resected stage III ovarian cancer: a Gynecologic Oncology Group study. J Clin Oncol 2003;21:3194-200.

3. Agarwal R, Kaye SB. Ovarian cancer: strategies for overcoming resistance to chemotherapy. Nat Rev Cancer 2003;3:502-16.

4. Mantia-Smaldone GM, Edwards RP, Vlad AM. Targeted treatment of recurrent platinum-resistant ovarian cancer: current and emerging therapies. Cancer Manag Res 2011;3:25-38.

5. Stordal B, Pavlakis N, Davey R. A systematic review of platinum and taxane resistance from bench to clinic: an inverse relationship. Can- cer Treat Rev 2007;33:688-703.

6. Steg AD, Bevis KS, Katre AA, Ziebarth A, Dobbin ZC, Alvarez RD, et al. Stem cell pathways contribute to clinical chemoresistance in ovarian cancer. Clin Cancer Res 2012;18:869-81 .

7. Ricci F, Bernasconi S, Perego P, Ganzinelli M, Russo G, Bono F, et al. Ovarian carcinoma tumor-initiating cells have a mesenchymal phenotype. Cell Cycle 2012;11:1966-76.

8. Yonemura Y, Kawamura T, Bandou E, Tsukiyama G, Endou Y, Miura $\mathrm{M}$. The natural history of free cancer cells in the peritoneal cavity. Recent Results Cancer Res 2007;169:11-23.

9. Chaffer CL, Weinberg RA. A perspective on cancer cell metastasis. Science 2011;331:1559-64.

10. Singh A, Settleman J. EMT, cancer stem cells and drug resistance: an emerging axis of evil in the war on cancer. Oncogene 2010;29: 4741-51.

11. Haslehurst AM, Koti M, Dharsee M, Nuin P, Evans K, Geraci J, et al. EMT transcription factors snail and slug directly contribute to cisplatin resistance in ovarian cancer. BMC Cancer 2012;12:91.

12. Ha M, Kim VN. Regulation of microRNA biogenesis. Nat Rev Mol Cell Biol 2014;15:509-24.

13. Sorrentino A, Liu CG, Addario A, Peschle C, Scambia G, Ferlini C. Role of microRNAs in drug-resistant ovarian cancer cells. Gynecol Oncol 2008;111:478-86.

14. Sui GQ, Fei D, Guo F, Zhen X, Luo Q, Yin S, et al. MicroRNA-338-3p inhibits thyroid cancer progression through targeting AKT3. Am J Cancer Res 2017;7:1177-87.

15. Cao R, Shao J, Hu Y, Wang L, Li Z, Sun G, et al. microRNA-338-3p inhibits proliferation, migration, invasion, and EMT in osteosarcoma cells by targeting activator of $90 \mathrm{kDa}$ heat shock protein ATPase homolog 1. Cancer Cell Int 2018;18:49.

16. Sun F, Yu M, Yu J, Liu Z, Zhou X, Liu Y, et al. miR-338-3p functions as a tumor suppressor in gastric cancer by targeting PTP1B. Cell Death Dis 2018;9:522.

17. Liu X, Wen J, Wang H, Wang Y. Long non-coding RNA LINC00460 promotes epithelial ovarian cancer progression by regulating microRNA-338-3p. Biomed Pharmacother 2018;108:1022-8.

18. Li Z, Hu S, Wang J, Cai J, Xiao L, Yu L, et al. MiR-27a modulates MDR1/P-glycoprotein expression by targeting HIPK2 in human ovarian cancer cells. Gynecol Oncol 2010;119:125-30.

19. Eoh KJ, Lee SH, Kim HJ, Lee JY, Kim S, Kim SW, et al. MicroRNA-630 inhibitor sensitizes chemoresistant ovarian cancer to chemotherapy by enhancing apoptosis. Biochem Biophys Res Commun 2018;497:513-20.

20. Zhang P, Zhu J, Zheng Y, Zhang H, Sun H, Gao S. miRNA-574-3p inhibits metastasis and chemoresistance of epithelial ovarian cancer (EOC) by negatively regulating epidermal growth factor receptor (EGFR). Am J Transl Res 2019;11:4151-65.

21. Li X, Chen W, Jin Y, Xue R, Su J, Mu Z, et al. miR-142-5p enhances cisplatin-induced apoptosis in ovarian cancer cells by targeting multiple anti-apoptotic genes. Biochem Pharmacol 2019;161:98112.

22. Boyerinas B, Park SM, Murmann AE, Gwin K, Montag AG, Zillhardt $\mathrm{M}$, et al. Let-7 modulates acquired resistance of ovarian cancer to Taxanes via IMP-1-mediated stabilization of multidrug resistance 1. Int J Cancer 2012;130:1787-97.

23. Zhang Y, Shi B, Chen J, Hu L, Zhao C. MiR-338-3p targets pyruvate kinase M2 and affects cell proliferation and metabolism of ovarian cancer. Am J Transl Res 2016;8:3266-73.

24. Jiang YC, Ma JX. The role of MiR-324-3p in polycystic ovary syndrome (PCOS) via targeting WNT2B. Eur Rev Med Pharmacol Sci 2018;22:3286-93.

25. Li G, Wang Y, Liu Y, Su Z, Liu C, Ren S, et al. miR-185-3p regulates nasopharyngeal carcinoma radioresistance by targeting WNT2B 
in vitro. Cancer Sci 2014;105:1560-8.

26. Zhang Y, Dun Y, Zhou S, Huang XH. LncRNA HOXD-AS1 promotes epithelial ovarian cancer cells proliferation and invasion by targeting miR-133a-3p and activating Wnt/ $\beta$-catenin signaling pathway. Biomed Pharmacother 2017;96:1216-21.

27. Arend RC, Londoño-Joshi AI, Straughn JM Jr, Buchsbaum DJ. The Wnt/ $\beta$-catenin pathway in ovarian cancer: a review. Gynecol On- col 2013;131:772-9.

28. Du B, Shim JS. Targeting epithelial-mesenchymal transition (EMT) to overcome drug resistance in cancer. Molecules 2016;21:965.

29. Vleminckx K, Vakaet L Jr, Mareel M, Fiers W, van Roy F. Genetic manipulation of E-cadherin expression by epithelial tumor cells reveals an invasion suppressor role. Cell 1991;66:107-19. 